\title{
ESTUDO DO PROCESSO DE EXTRAÇÃO DOS COMPOSTOS FENÓLICOS E ANTOCIANINAS TOTAIS DO HIBISCUS SABDARIFFA
}

\author{
M. GUINDANI ${ }^{1}$, F. TONET ${ }^{1}$, F. KUHN ${ }^{1}$, J. DAL MAGRO ${ }^{2}$, F. DALCANTON ${ }^{2}$, M. A. FIORI ${ }^{2}$, \\ J. M. M. MELLO ${ }^{2}$ \\ ${ }^{1}$ Universidade Comunitária da Região de Chapecó, Área de Ciências Exatas e Ambientais \\ ${ }^{2}$ Universidade Comunitária da Região de Chapecó, Programa de Pós-Graduação em Ciências \\ Ambientais e Programa de Pós-Graduação em Tecnologia e Gestão da Inovação \\ E-mail para contato: guindanimarcelo@unochapeco.edu.br
}

\begin{abstract}
RESUMO - Atualmente, busca-se por benefícios provenientes dos produtos naturais terapêuticos, dentre eles destaca-se o hibisco, rico em antocianinas e outros compostos fenólicos responsáveis pelo caráter antioxidante. Neste sentido, este trabalho teve por objetivo avaliar as melhores condições de extração de compostos fenólicos de flores do Hibiscus sabdariffa. As flores foram maceradas em diferentes sistemas de solventes que compreendem: água acidificada ( $\mathrm{pH}$ 1,5), misturas de 50, 70, 90 e 100\% (v/v) etanol/água e misturas de 50, 70, 90 e 100\% (v/v) metanol/água. O teor de compostos fenólicos totais foi analisado pelo método espectrofotométrico de Folin-Ciocalteu, utilizando ácido gálico como padrão, e o método $\mathrm{pH}$ diferencial foi usado para as antocianinas monoméricas. A partir das diferentes condições de extração analisadas, observou-se uma melhor resposta com o metanol 100\% para os compostos fenólicos $(4825,00 \mathrm{mg} / \mathrm{L})$ e metanol/água 70/30 para as antocianinas $(928,46 \mathrm{mg} / \mathrm{L})$.
\end{abstract}

\section{INTRODUÇÃO}

O mercado mundial tem apresentado um crescente interesse, em ofertar alimentos com características bioativas, contendo substâncias que influenciam nas atividades fisiológicas ou metabólicas, contribuindo para uma melhor qualidade de vida das populações. Tais alimentos são conhecidos atualmente como "alimentos funcionais" (Vieira et al., 2010). Estes alimentos apresentam atividades biológicas e nutricionais. Ademais, demonstram capacidade de regular as funções corporais, auxiliando na proteção contra doenças como hipertensão, diabetes, câncer, entre outras (Souza et al., 2007).

O Hibiscus sabdariffa, conhecido popularmente como hibisco, é uma planta medicinal utilizada principalmente como hipertensivo e redutor de colesterol. Por ser rico em antocianinas, vitamina C, licopeno, betacaroteno e polifenóis, possui atividade antioxidante no sequestro de radicais livres, de modo a auxiliar no tratamento de doenças degenerativas. O interesse econômico do hibisco está nos cálices desidratados, utilizados mundialmente para a produção de bebidas, alimentos, conservantes e antioxidantes (Maciel, 2011).

Em busca de mais alimentos ricos em compostos fenólicos e consequentemente com alta atividade antioxidante, a indústria alimentícia já se utiliza dos benefícios do $H$. sabdariffa, tanto de suas folhas quanto do cálice, que é a parte da corola composta pelas pétalas de intensa coloração 


\section{9 a 22 de outubro de 2014 \\ Florianópolis/SC}

vermelha. Os compostos fenólicos são substâncias que existem principalmente nas plantas, como é o caso do hibisco, mas que podem também ser provenientes do catabolismo dos aminoácidos. A importância dada a estes compostos deve-se ao fato de muitos possuírem propriedades benéficas para a saúde humana (Marques et al., 2004). Os compostos fenólicos apresentam múltiplas atividades biológicas, tais como: propriedades anti-tumorais, anti-mutagénicas, anti-inflamatórias, antibacterianas e antioxidantes, por protegerem as células contra os danos oxidativos (Sousa, 2008).

Além disso, as antocianinas são uma classe de compostos fenólicos que representam um significante papel na prevenção ou retardo do aparecimento de várias doenças por suas propriedades antioxidantes. Seu espectro de cor vai do vermelho ao azul, apresentando-se também como uma mistura de ambas as cores resultando em tons de púrpura. Muitas frutas, hortaliças e flores devem sua atrativa coloração a esses pigmentos que se encontram dispersos nos vacúolos celulares (Volp et al., 2008).

A extração é um dos processos mais utilizados para o isolamento de produtos ativos presentes em uma planta medicinal. Dentre os métodos de extração sólido-líquido mais empregados, destacamse os convencionais de maceração, percolação e extração com Soxhlet; e os não convencionais: ultrasom, extração com fluido supercrítico e extração com líquido pressurizado (Melecchi, 2005). Eficientes métodos de extração devem maximizar a recuperação de compostos alvo, tais como antocianinas, com quantidade mínima de degradação ou alteração de seu estado natural. E este trabalho teve por objetivo avaliar algumas condições de extração dos compostos fenólicos e antocianinas totais variando o tipo de solvente bem como sua composição.

\section{MATERIAL E MÉTODOS}

\subsection{Materiais}

As flores de Hibiscus sabdariffa, foram adquiridas junto ao Laboratório Industrial Vida e Saúde LTDA, localizado na cidade de Chapecó (SC), no mês de novembro de 2013. Todos os reagentes químicos utilizados tiveram grau analítico de pureza.

\subsection{Extração dos Extratos}

Os compostos fenólicos bem como as antocianinas totais foram extraídos utilizando $10 \mathrm{~g} \mathrm{de}$ amostra seca, colocadas em erlenmeyers e adicionando-se $50 \mathrm{~mL}$ de solvente. Os solventes utilizados foram água acidificada ( $\mathrm{pH}$ 1,5), metanol e etanol. As proporções foram obtidas da seguinte forma: água acidificada com $\mathrm{HCl}(\mathrm{pH}$ 1,5); etanol/água nas proporções de 50, 70, 90 e $100 \%$ (v/v); e metanol/água também nas proporções de 50, 70, 90 e $100 \%$ (v/v). O tempo de extração utilizado foi de $24 \mathrm{~h}$ mantendo-se a temperatura constante de $25^{\circ} \mathrm{C}$. Posteriormente, filtrou-se por gravidade o extrato para a separação do material sólido. Todos os experimentos foram realizados em triplicata.

\subsection{Determinação dos Compostos Fenólicos Totais}

A concentração de compostos fenólicos totais foi determinada pelo método de Folin-Ciocaulteu, conforme metodologia proposta por Shahidi e Naczk (1995). Para a construção da curva analítica partiu-se da solução padrão de ácido gálico em diferentes concentrações de $\mathrm{mg} / \mathrm{L}$. Para determinar a concentração dos compostos fenólicos (nas soluções padrão e amostras), retirou-se uma alíquota de 0,5 $\mathrm{mL}$, estes foram transferidas para tubos de ensaio protegidos da luz com papel alumínio, em seguida, 


\section{9 a 22 de outubro de 2014 \\ Florianópolis/SC}

adicionou-se 3,5 mL de água destilada e $0,25 \mathrm{~mL}$ do reagente Folin Ciocalteau. A solução foi homogeneizada com o auxílio de um agitador de tubos de ensaio Vortex, e após 3 minutos, acrescentou-se $0,75 \mathrm{~mL}$ de solução saturada de $\mathrm{Na}_{2} \mathrm{CO}_{3}$ e deixou-se por uma hora em repouso ao abrigo da luz. Após, realizou-se as leituras das absorbâncias em espectrofotômetro UV-visível à 765 nm (modelo SP 220, marca Bioespectro). Para o branco adicionou-se todos os reagentes menos as soluções padrão ou as amostras de hibisco.

Através da equação da curva de calibração e com os valores das absorbâncias das amostras, realizou-se o cálculo do teor de compostos fenólicos totais, expresso em mg de ácido gálico/100 g de amostra.

\subsection{Determinação das Antocianinas Totais}

A determinação de antocianinas totais foi realizada pelo método do $\mathrm{pH}$ diferencial (Giusti e Wrolstad, 2001), no qual foram utilizados dois sistemas tampão: ácido clorídrico/cloreto de potássio de pH 1,0 (0,025 M) e ácido acético/acetato de sódio de pH 4,5 $(0,4 \mathrm{M})$. Com 0,2 $\mathrm{mL}$ de uma amostra diluída do extrato (para conseguir uma absorbância na faixa de 0,10-1,20 a $510 \mathrm{~nm}$ ) adicionou-se 1,8 $\mathrm{mL}$ da correspondente dissolução tampão e mediu-se a absorbância frente a um branco a $520 \mathrm{~nm}$ e 700 nm. Calculou-se a absorbância final da amostra, A, através da Equação 1.

$$
A=\left(A_{\text {max.vis }}-A_{700 n m}\right) p H 1,0-\left(A_{\text {max.vis }}-A_{700 n m}\right) p H 4,5
$$

E pela Equação 2 foi possível realizar o cálculo da concentração das antocianinas totais monoméricas.

Pigmento de antocianina monomérica $(m g / L)=(A . P M . D F .1000) /(\varepsilon .1)$

Onde: $P M$ é o peso molecular da antocianina majoritária no solvente específico; $D F$ é o fator de diluição (30); e $\varepsilon$ é a absortividade molar para o pigmento majoritário da amostra no solvente específico $(\varepsilon=29000)$.

Os resultados de compostos fenólicos e antocianinas realizados em triplicata foram analisados estatisticamente utilizando o software Statistica 7.0 (StatSoft ${ }^{\circledR}$ ), através da análise de variância (ANOVA) pelo teste de Tukey com 5\% de probabilidade, ou seja, diferenças significativas apresentam $\mathrm{p} \leq 0,05$.

\section{RESULTADOS E DISCUSSÃO}

Os resultados para a extração dos compostos fenólicos após $24 \mathrm{~h}$ e seus respectivos desvios, estão representados na Figura 1 e na Tabela 1 para todas as condições estudadas. 


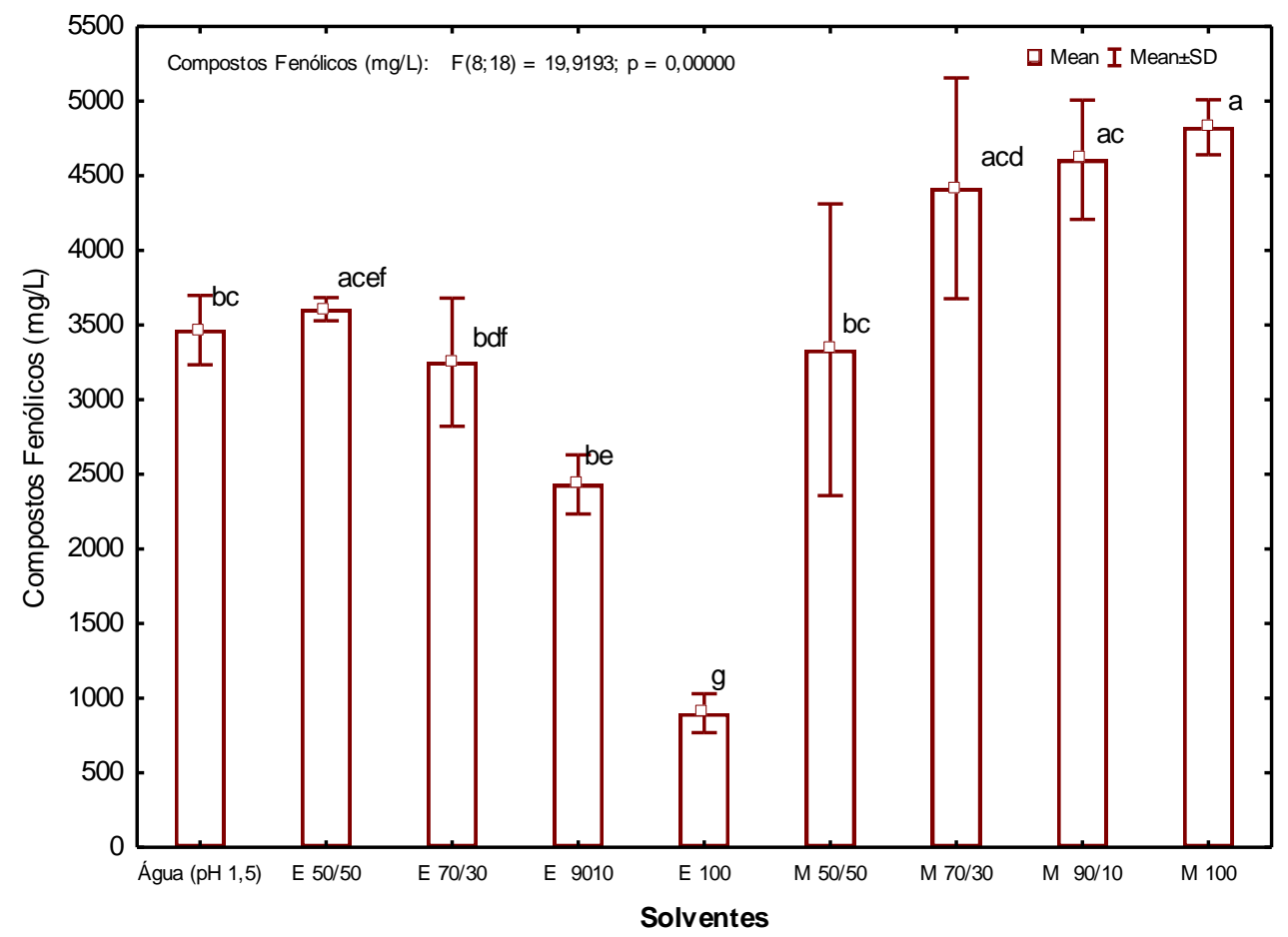

Figura 1 - Concentração dos compostos fenólicos, para as nove condições de extração estudadas.

Tabela 1 - Valor médio e desvio padrão da concentração de compostos fenólicos nos diferentes meios de extração

\begin{tabular}{lc}
\hline Solventes & Compostos Fenólicos $(\mathbf{m g} / \mathbf{L})$ \\
\hline Água pH 1,5 & $3466,30 \pm 232,56^{\mathrm{bc}}$ \\
Etanol 50/50 & $3605,80 \pm 77,31^{\mathrm{acef}}$ \\
Etanol 70/30 & $3250,73 \pm 429,32^{\mathrm{bdf}}$ \\
Etanol 90/10 & $2431,88 \pm 198,38^{\mathrm{be}}$ \\
Etanol 100 & $899,28 \pm 130,59^{\mathrm{g}}$ \\
Metanol 50/50 & $3334,06 \pm 976,99^{\mathrm{bc}}$ \\
Metanol 70/30 & $4415,58 \pm 739,32^{\mathrm{acd}}$ \\
Metanol 90/10 & $4607,61 \pm 399,86^{\mathrm{ac}}$ \\
Metanol 100 & $4825,00 \pm 184,06^{\mathrm{a}}$ \\
\hline
\end{tabular}

Médias seguidas de mesma letra na coluna não diferem entre si pelo teste de Tukey a 5\% de probabilidade $(\mathrm{p}<0,05 \%)$.

Através da Figura 1 e da Tabela 1, pode-se observar que a melhor condição de extração de compostos fenólicos foi para o metanol $100 \%(4825,00 \mathrm{mg} / \mathrm{L})$, porém estatisticamente não há diferença significativa na concentração dos compostos fenólicos se for utilizado 90/10 ou 70/30 de metanol/água $(4607,61 \mathrm{mg} / \mathrm{L}$ e 4415,58 mg/L, respectivamente). Verifica-se também que a menor concentração de compostos fenólicos obtida foi para a condição $100 \%$ etanol $(899,28 \mathrm{mg} / \mathrm{L})$. Para a água acidificada $(3466,30 \mathrm{mg} / \mathrm{L})$, observa-se que há diferença significativa quando comparado ao metanol 100\%, mas quando comparado a solução metanol/água 90/10 ou 70/30, não há diferença, o que permite utilizar esse solvente para extrair compostos fenólicos com possível aplicação na área alimentícia ou farmacêutica levando em consideração que este sistema de solvente não é tóxico quando 
comparado aos sistemas que possuem metanol na composição. Pode-se dizer que através dos solventes com características mais polares, neste caso, o metanol, obtém-se maior eficiência de extração de compostos fenólicos, por outro lado, a extração utilizando-se $100 \%$ etanol, o qual possui caráter mais apolar quando comparado ao metanol, mostrou-se menos eficiente frente as demais condições.

A Figura 2 e a Tabela 2 apresentam os resultados da extração de antocianinas (mg $\backslash \mathrm{L})$ para as nove condições diferentes de solventes.

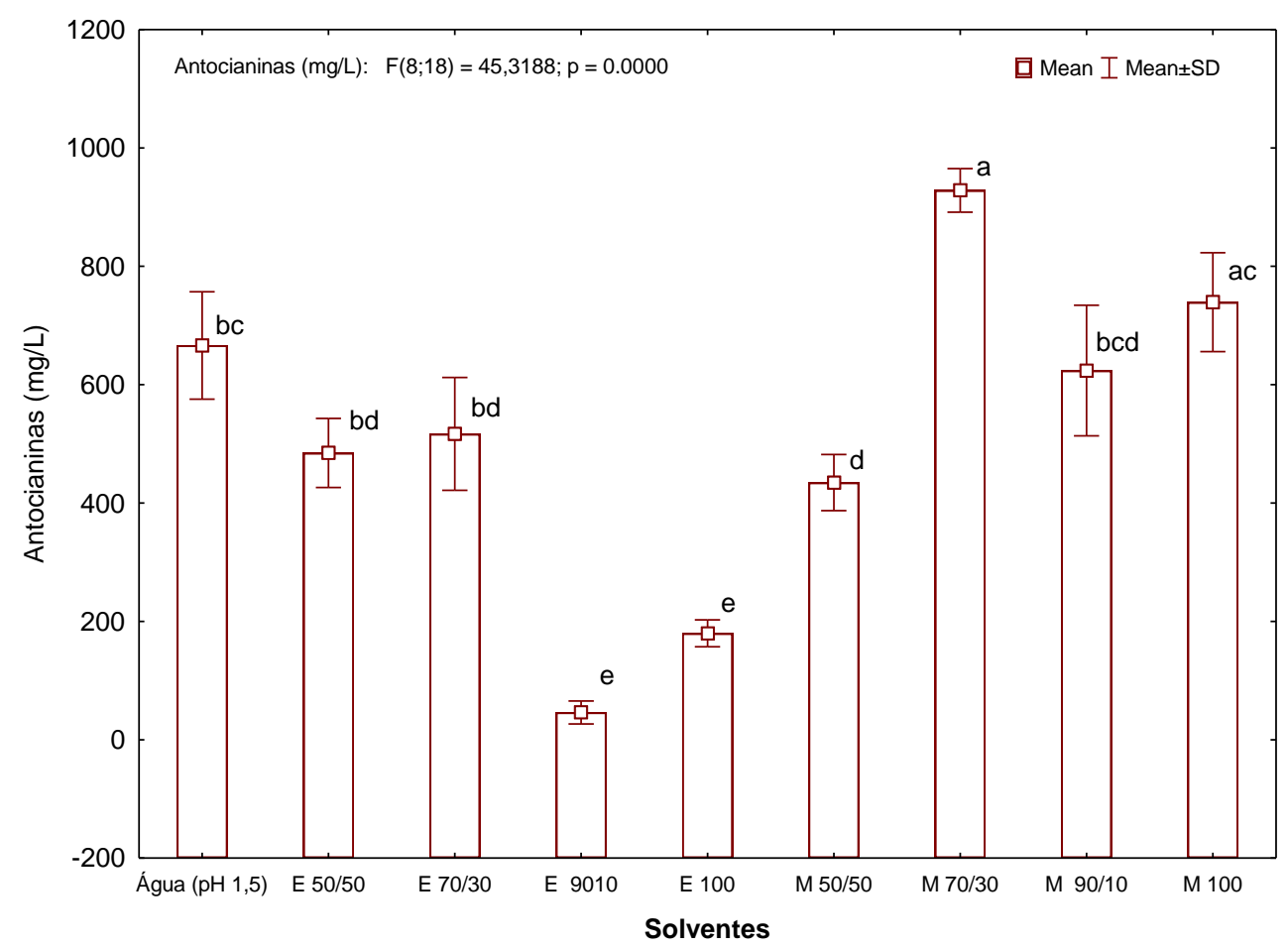

Figura 2 - Concentração de antocianinas extraída através dos nove diferentes meios de extração.

Tabela 2 - Valor médio e desvio padrão da concentração de antocianinas nos diferentes meios de extração

\begin{tabular}{lc}
\hline Solventes & Antocianinas $(\mathbf{m g} / \mathbf{L})$ \\
\hline Água pH 1,5 & $666,29 \pm 90,69^{\mathrm{bc}}$ \\
Etanol 50/50 & $484,60 \pm 58,40^{\mathrm{bd}}$ \\
Etanol 70/30 & $516,83 \pm 95,32^{\mathrm{bd}}$ \\
Etanol 90/10 & $46,09 \pm 19,33^{\mathrm{e}}$ \\
Etanol 100 & $179,85 \pm 22,63^{\mathrm{e}}$ \\
Metanol 50/50 & $434,67 \pm 47,37^{\mathrm{d}}$ \\
Metanol 70/30 & $928,46 \pm 36,83^{\mathrm{a}}$ \\
Metanol 90/10 & $623,87 \pm 110,25^{\mathrm{bcd}}$ \\
Metanol 100 & $739,45 \pm 83,63^{\mathrm{ac}}$ \\
\hline
\end{tabular}

Médias seguidas de mesma letra na coluna não diferem entre si pelo teste de Tukey a 5\% de probabilidade $(\mathrm{p}<0,05 \%)$.

Pela análise da Figura 2 e da Tabela 2, observa-se que a condição que extraiu a maior concentração de antocianinas foi metanol/água 70/30 (928,46 mg/L), porém essa condição não 
apresenta diferença estatística significativa com a condição $100 \%$ metanol (739,43 mg/L). A segunda melhor condição de extração foi a água acidificada $(666,29 \mathrm{mg} / \mathrm{L})$, conforme já comentado, apresenta como benefícios sua aplicação em alimentos ou fármacos. A menor concentração de antocianinas foi obtida para a condição $90 / 10$ de etanol/água $(46,09 \mathrm{mg} / \mathrm{L})$, e esta não apresentou diferença significativa quando comparada a condição $100 \%$ etanol $(179,85 \mathrm{mg} / \mathrm{L})$. Pode-se dizer também que quanto mais polar for o solvente, maior a concentração de antocianinas obtidas no extrato, e a medida que sua polaridade vai reduzindo, a extração de antocianinas é prejudicada. As antocianinas apresentam como estrutura fundamental o cátion flavílico (Patil et al. 2009), bem como o açúcar presente na molécula (Figura 3) confere um caráter hidrofílico, o que sugere que para a extração de antocianinas a água seja um bom solvente.<smiles>CCOc1cc2c(O)cc(O)cc2[o+]c1-c1ccc(O)c(O)c1</smiles>

Figura 3 - Estrutura da cianidina 3-glucosídeo, antocianina presente na maioria dos vegetais.

Teixeira et al. (2008) utilizaram uma solução etanol/água 70/30 acidificada para a análise de antocianinas presentes no Hibiscus rosa-sinensis, com um tempo de extração de $24 \mathrm{~h}$, seu resultado de concentração $250,97 \mathrm{mg} / 100 \mathrm{~g}$. Chandrasekhar et al. (2012), estudaram diferentes solventes para extrair antocianinas de repolho roxo, e a melhores condições observadas foram acetona ( $\mathrm{pH} \mathrm{3,65)}$ $(426 \mathrm{mg} / \mathrm{L})$ e $1 \% \mathrm{HCl}$ em metanol $(420 \mathrm{mg} / \mathrm{L})$. Valores esses semelhantes aos encontrados neste trabalho.

\section{CONCLUSÃO}

A partir dos resultados de extração de compostos fenólicos e antocianinas do hibisco é possível observar as diferenças entre os nove tipos de solventes utilizados nas extrações, sendo que dentre os meios estudados, a maior concentração de compostos fenólicos obtido foi com a utilização do metanol $100 \%$, sendo este de 4825,00 mg/L. Para as antocianinas, a melhor extração ocorreu com o metanol/água 70/30, sendo esse de 928,46 mg/L . Deve-se ressaltar que pesquisas objetivando a quantificação de compostos fenólicos e antocianinas deveriam ocorrer com mais frequência, devido aos seus benefícios já comprovados, onde o Hibiscus sabdariffa destaca-se devido as suas consideráveis quantidades destas substâncias.

\section{AGRADECIMENTO}

Os autores agradecem o auxílio financeiro do Programa Institucional de Bolsas em Desenvolvimento Tecnológico e Inovação (PIBITI), do Conselho Nacional de Desenvolvimento Científico e Tecnológico (CNPq), e ao Laboratório Industrial Vida e Saúde LTDA que disponibilizou a matéria-prima.

\section{REFERÊNCIAS}


CARVALHO, L. M.; SILVA, R. B.; SILVA, M. M.; CARCARÁ, V, A. K.; AMORIM, R.R. Estudo comparativo entre a quantidade de fenólicos totais presentes em folhas e cálices de Hibiscus sabdariffa L. VII CONEEPI - Congresso Norte Nordeste de Pesquisa e Inovação. Tocantins, 2012.

CHANDRASEKHAR, J.; MADHUSUDHAN, M. C.; RAGHAVARAO K. S. M. S. Extraction of anthocyanins from red cabbage and purification using adsorption. Food Bioprod. Process. v. 90, p. 615-623,

MACIEL, M. J. Avaliação do extrato alcoólico de hibisco (hibiscus sadbariffa L.) como fato de proteção antibacteriana e antioxidante em alimentos. 2011. 61f. Tese (dissertação em tecnologia de alimentos) - Instituto de ciencia e tecnologia de alimentos, Universidade federal do Rio Grande do Sul, Porto Alegre, 2011.

MARQUES, C.; DUTHOIT, M.; ALVARENGA, B.; ROSEIRO, L. B. Implementação e validação do método de Folin-Ciocalteu para a determinação do teor em compostos fenólicos totais - aplicação a extratos de flor de cardo e queijo. Tese de Licenciatura, Instituto Politécnico de Beja. Beja, 2004.

MELECCHI, S. I. M. Caracterização química de extratos de Hibiscus tiliaceus L: Estudo comparativo de métodos de extração. 2005. 197f. Tese (Doutorado em Química), Universidade Federal do Rio Grande Do Sul, Porto Alegre, 2005.

SOUSA, J. B. A. S. P. Atividade Biológica de derivados do Ácido Cafeíco: Efeito antioxidante e antiinflamatório. Dissertação apresentada para a obtenção do grau de Mestre em Controle de Qualidade. Universidade do Porto. Porto, 2008.

SOUZA, L. G., MENDES, J. U. L., NETO, H. J., SANTOS, R. G., MELO, A. V., Obtenção de tomates secos utilizando um sistema de secagem solar construídos com materiais alternativos. $8^{o}$ Congresso Iberoamericano de engenharia mecânica. Cusco, 2007.

PATIL, G.; MADHUSUDHAN, M. C.; RAVINDRA, B.; RAGHAVARAO, K. S. M. S. Extraction, dealcoholisation and concentration of anthocyanin from red radish peels. Chem. Eng. Processing, p. 364-369, 2009.

SHAHIDI, F.; NACZK, M. Food phenolics: souces, chemistry, effects and applications. 1 ed. Lancaster: Technomic Publishing Co,Inc., 1995. 331p

TEIXEIRA, N. L.; STRINGHETA, P. C; ALVES, O. F. Comparação de métodos para quantificação de antocianinas. Revista Ceres, v. 55, p. 297-304, 2008.

VIEIRA, A. C. P.; CORNÉLIO, A. R.; SALGADO, J. M. Alimentos funcionais: aspectos relevantes para o consumidor. Disponível em: <http://www.jus.com.br/doutrina/texto.asp?id=8702>. Acesso em: 01 de setembro de 2013.

VOLP, P. C. A.; RENHE, T. R. I.; BARRA. K.; STRINGUETA, C. P. Flavonóides antocianinas: características e propriedades na nutrição e saúde. Revista Brasileira de Nutrição. Minas Gerais, 2008. 